\title{
Special functions and perturbations of black holes
}

\author{
E. G. Kalnins \\ Department of Mathematics, University of Waikato, \\ Hamilton, New Zealand. \\ W. Miller Jr. \\ School of Mathematics, University of Minnesota, \\ Minneapolis, Minnesota 55455, U.S.A. \\ G. F. Torres del Castillo \\ Departamento de Fisica Matematica, \\ Instituto de Ciencas de la Universidad Autonoma Puebla \\ Puebla, Mexico. \\ and G. C. Williams \\ Department of Mathematics, University of Waikato, \\ Hamilton, New Zealand.
}

December 23, 1999

\begin{abstract}
It is known that perturbations of black holes for which not all of the defining parameters (i.e., mass, angular momentum and charge) are nonzero can be computed using Debye potentials, which are special functions of confluent Heun type. There is however no scheme for the corresponding solution of the perturbation problem for a massive charged rotating black hole. In this paper we discuss how this problem may be solved using the idea of a symmetry operator and an integral equation formulation. In addition we give a summary of the geometric features of the black hole spacetimes which account for some of their remarkable properties.
\end{abstract}




\section{Special functions and perturbations of mas- sive rotating charged black holes}

The behaviour of space times which describe the geometry of black holes when subject to perturbations is interesting from both the mathematical and physical point of view. Black holes are solutions of Einstein's field equations which are completely characterised by three numbers that can be identified with mass, charge and angular momentum. The analysis of perturbations of black hole space times with some of their parameters zero can be found in the work of Chandrasekhar [1], and Cohen and Kegeles [2]. One of the motivations for studying perturbations of space times is to obtain some idea of the stability of the space times subject to small changes in the geometry, i.e., the metric tensor. Another consideration is the scattering of small perturbing particles in the presence of the curved spacetime of a black hole. The original observation that perturbations of black holes were amenable to explicit treatment goes back to Teukolsky [3] who demonstrated that the first order perturbed part of the Weyl tensor (i.e., a combination of the curvature tensor, Ricci tensor and Ricci scalar) satisfied a second order linear partial differential equation that was solvable by the method of separation of variables.

The subject of perturbations is also closely associated with the problem of solution of wave equations in a curved spacetime background. In fact, gravitational perturbations can be regarded as the equations for a spin 2 field in the case of no charge. For the well known equations of Dirac and Maxwell, explicit solutions can be obtained in a black hole spacetime background [4]. In this article we discuss the nature of the perturbation problem for a charged rotating black hole,indicating the essential differences with the previously known cases. This problem was initially studied by Chandrasekhar but has as yet not been comprehensively solved.

The most general infinitesmal distance or line element of a charged rotating black hole is given by the Kerr Newman black hole metric

$$
d s^{2}=\bar{\rho} \bar{\rho}^{*}\left(\frac{d r^{2}}{\Delta}+d \theta^{2}\right)+\left(r^{2}+a^{2}\right) \sin ^{2} \theta d \phi^{2}-d t^{2}+\frac{2 M r}{\bar{\rho} \bar{\rho}^{*}}\left(a \sin ^{2} \theta d \phi-d t\right)^{2}
$$

where $\bar{\rho}=r+i a \cos \theta$ and $\Delta=r^{2}+a^{2}+q^{2}-2 M r$. 
This metric describes a charged rotating black hole of mass $M$, charge $q$ and angular momentum $J=a M$. The associated vector potential for the electromagnetic field has nonzero components $A_{t}=q r, A_{\phi}=-a q r \sin ^{2} \theta$. The identification of these parameters in classical terms is achieved by considering the leading terms in the metric asymptotically, i.e., for large $r$. In order to make computation easier it is convenient to choose the coordinates $t, r, \theta, \phi$ as in (1) and also important to choose a tetrad of vectors (i.e., a moving frame of reference) located at each point. It is convenient to choose these vectors $l, n, m$ and $m^{*}$ in such a way that they constitute a null tetrad for which the only nonzero inner products are

$$
l \cdot n=1, \quad m \cdot m^{*}=-1 .
$$

An important choice in the case of the Kerr Newman black hole is given by

$$
\begin{gathered}
l^{i}=\frac{1}{\Delta}\left(r^{2}+a^{2}, \Delta, 0, a\right), \quad n^{i}=\frac{1}{2 \rho^{2}}\left(r^{2}+a^{2},-\Delta, 0, a\right), \\
m^{i}=\frac{1}{\sqrt{2} \bar{\rho}}(i a \sin \theta, 0,1, i \csc \theta), \quad m^{* i}=\frac{1}{\sqrt{2} \bar{\rho}^{*}}(-i a \sin \theta, 0,1,-i \csc \theta),
\end{gathered}
$$

using in order the coordinates $t, r, \theta, \varphi$. Associated with these vectors are the directional derivatives

$$
\begin{gathered}
D=l^{i} \partial_{i}=\frac{1}{\Delta}\left(\left(r^{2}+a^{2}\right) \partial_{t}+\Delta \partial_{r}+a \partial_{\varphi}\right), \Delta=n^{i} \partial_{i}=\frac{1}{2 \rho^{2}}\left(\left(r^{2}+a^{2}\right) \partial_{t}-\Delta \partial_{r}+a \partial_{\varphi}\right) \\
\delta=m^{i} \partial_{i}=\frac{1}{\sqrt{2} \bar{\rho}}\left(i a \sin \theta \partial_{t}+\partial_{\theta}+i \csc \theta \partial_{t}\right) \\
\delta^{*}=m^{* i} \partial_{i}=\frac{1}{\sqrt{2} \bar{\rho}^{*}}\left(-i a \sin \theta \partial_{t}+\partial_{\theta}-i \csc \theta \partial_{t}\right) .
\end{gathered}
$$

It is very convenient to refer all tensor quantities to the tetrad of vectors we have adopted. In these terms the basic contribution of Teukolsky was to demonstrate that the first order perturbed part of the component $\Psi_{0}=$ $-C_{p q r s} l^{p} m^{q} l^{r} m^{s}$ of the Weyl tensor when written in the form $\Psi_{0}=\Phi_{0} e^{i(m \phi+\sigma t)}$ satisfies

$$
\left(\Delta D_{1,0,0} D_{2,0,0}^{+}+L_{-1,0,0} L_{2,0,0}^{+}-6 i \sigma(r+i a \cos \theta)\right) \Phi_{0}=0 .
$$


Here $C_{p q r s}$ is the Weyl tensor expressed in terms of the Riemann curvature tensor and its contractions via

$$
C_{p q r s}=R_{p q r s}-\frac{1}{2}\left(g_{p r} R_{q s}+g_{q s} R_{p r}-g_{q r} R_{p s}-g_{p s} R_{q r}\right)+\frac{1}{6}\left(g_{p r} g_{q s}-g_{p s} g_{q r}\right) R,
$$

(see for instance Eisenhart [5]) where

$$
\begin{aligned}
D_{c_{1}, c_{2}, c_{3}}= & \partial_{r}+\frac{i K+2 c_{1}(r-M)}{\Delta}+\frac{c_{2}}{\bar{\rho}^{*}}+\frac{c_{3}}{\bar{\rho}}, \quad K=\left(r^{2}+a^{2}\right) \sigma+a m, \\
D_{c_{1}, c_{2}, c_{3}}^{+}= & \partial_{r}-\frac{i K+2 c_{1}(r-M)}{\Delta}+\frac{c_{2}}{\bar{\rho}^{*}}+\frac{c_{3}}{\bar{\rho}}, \quad Q=a \sigma \sin \theta+m \csc \theta \\
& L_{c_{1}, c_{2}, c_{3}}=\partial_{\theta}+Q+c_{1} \cot \theta+i a \sin \theta\left(\frac{c_{2}}{\bar{\rho}^{*}}+\frac{c_{3}}{\bar{\rho}}\right), \\
& L_{c_{1}, c_{2}, c_{3}}^{+}=\partial_{\theta}-Q+c_{1} \cot \theta+i a \sin \theta\left(\frac{c_{2}}{\bar{\rho}^{*}}+\frac{c_{3}}{\bar{\rho}}\right) .
\end{aligned}
$$

The equation for $\Phi_{0}$ has solutions of the form $\Phi_{0}=R_{2}(r) S_{2}(\theta)$. The explicit form of the separation equations is

$$
\begin{gathered}
{\left[\Delta \partial_{r}^{2}+6(r-M) \partial_{r}+\frac{K^{2}-4 i K(r-M)}{\Delta}+8 i \sigma r-\lambda\right] R_{2}=0,} \\
{\left[\partial_{\theta}^{2}+\cot \theta \partial_{\theta}-a^{2} \sigma^{2} \sin ^{2} \theta-\frac{m^{2}+4 m \cos \theta+4}{\sin ^{2} \theta}-4 a \sigma \cos \theta+\lambda-2 a m \sigma+8\right] S_{2}=0 .}
\end{gathered}
$$

This equation for $S_{2}(\theta)$ is identifiable as a confluent Heun equation $[6,7]$ and is clearly a modified form of the spheroidal equation encountered in the classical separation of variables for the Helmholtz equation. The equation for $R_{2}(r)$ can be similarly identified. Here $S_{2}, R_{2}$ are Teukolsky functions of spin 2 [1]. The most transparent way to obtain gravitational perturbations for a black hole with rotation, mass and no charge has been given by Cohen and Kegeles [2]. These authors showed that the perturbed metric tensor is given by

$$
\begin{gathered}
h_{\mu \nu}=-\left\{l_{\mu} l_{\nu}\left(\delta^{*}+\alpha+3 \beta^{*}-\tau^{*}\right)\left(\delta^{*}+4 \beta^{*}+3 \tau^{*}\right)+m_{\mu}^{*} m_{\nu}^{*}\left(D-\rho^{*}\right)\left(D+3 \rho^{*}\right)\right. \\
\left.-l_{(\mu} m_{\nu)}\left[\left(D+\rho-\rho^{*}\right)\left(\delta^{*}+4 \beta^{*}+3 \tau^{*}\right)+\left(\delta^{*}-\alpha+3 \beta^{*}-\pi-\tau^{*}\right)\left(D+3 \rho^{*}\right)\right]\right\} R_{-2} S_{2} e^{i(\sigma t+m \varphi)} \\
+ \text { c.c. (complex conjugate). }
\end{gathered}
$$


Here we have used the so called spin coefficents in the Penrose notation, viz.

$$
\begin{gathered}
\rho=\frac{-1}{\bar{\rho}^{*}}, \quad \pi=\frac{i a \sin \theta}{\sqrt{2} \bar{\rho}^{* 2}}, \quad \tau=\frac{-i a \sin \theta}{\sqrt{2} \bar{\rho} \bar{\rho}^{*}}, \quad \mu=\frac{-\Delta}{2 \bar{\rho} \bar{\rho}^{* 2}}, \quad \gamma=\mu+\frac{(r-M)}{2 \bar{\rho} \bar{\rho}^{*}}, \\
\beta=\frac{\cot \theta}{2 \sqrt{2} \bar{\rho}}, \quad \alpha=\pi-\beta^{*} .
\end{gathered}
$$

The function $R_{-2} S_{2} e^{i(\sigma t+m \varphi)}$ is the Debye potential from which the metric is calculated by successive derivatives. In general, Teukolsky functions of spin $s$ are defined by the ordinary differential equations

$$
\begin{gathered}
\left(\Delta D_{1-s, 0,0}^{+} D_{0,0,0}+2(2 s-1) i \sigma r\right) R_{-s}=\lambda_{s} R_{-s}, \\
\left(\Delta D_{1,0,0} D_{s, 0,0}^{+}-2(2 s-1) i \sigma r\right) R_{s}=\lambda_{s} R_{s}, \\
\left(L_{1-s, 0,0}^{+} L_{s, 0,0}+2(2 s-1) \sigma a \cos \theta\right) S_{s}=-\lambda_{s} S_{s}, \\
\left(L_{1-s, 0,0} L_{s, 0,0}^{+}-2(2 s-1) \sigma a \cos \theta\right) S_{-s}=-\lambda_{s} S_{-s} .
\end{gathered}
$$

For each integer $s$ the spectrum of eigenvalues $\lambda_{s, n}, n=1,2,3 \cdots$ is discrete and countable. In the case of a charged black hole with no rotation the corresponding perturbation problem can be solved by similar means. In the work of Chandrasekhar [1] this problem is solved by a different method which is, however, equivalent to the Debye potential method. The advantage of zero rotation or angular momentum is that the angular dependence of the perturbations can be removed, since the equations are rotationally invariant. The main problem that remains in the perturbation theory of black holes is the so called Kerr Newman perturbation problem. This is the case in which the black hole has nonzero mass, angular momentum and charge. The formulation of the problem in terms of an analogue of Debye potential functions has been given by Torres del Castillo [8]. He showed the following. Suppose the functions $\Psi_{E}, \Psi_{G}, M_{1^{\prime}}$ and $M_{0^{\prime}}$ satisfy the differential equations

$$
\begin{gathered}
\left(\Delta+3 \rho-\rho^{*}\right) M_{1^{\prime}}-\left(\delta+3 \beta-\alpha^{*}+3 \tau+\pi^{*}\right) M_{0^{\prime}}=4 \varphi_{1} \Psi_{E} \\
\left(\delta^{*}+3 \alpha+\beta^{*}-\tau^{*}\right) M_{1^{\prime}}-\left(\Delta+3 \gamma-\gamma^{*}+\mu^{*}\right) M_{0^{\prime}}=2 \varphi_{1} \Psi_{G} \\
2 \varphi_{1}\left[\left(\delta^{*}+2 \alpha+\pi\right) \Psi_{E}-(D+2 \rho) \Psi_{G}\right]=\Omega_{+} M_{1^{\prime}} \\
2 \varphi_{1}\left[(\Delta+2 \gamma+\mu) \Psi_{E}-(\delta+4 \beta+2 \tau) \Psi_{G}\right]=\Omega_{-} M_{0^{\prime}}
\end{gathered}
$$


where $\Omega_{ \pm}=3 \Psi_{2} \pm 2 \Phi_{11}$,

$$
\Psi_{2}=-C_{p q r s} l^{p} m^{q} m^{r *} n^{s}=\frac{-M}{\bar{\rho}^{* 3}}+\frac{q^{2}}{\bar{\rho} \bar{\rho}^{* 3}}, \quad \phi_{1}=\frac{1}{2} F_{i j}\left(l^{i} n^{j}+m^{* i} m^{j}\right)=\frac{-q}{2 \bar{\rho}^{* 2}},
$$

and $\Phi_{11}=\phi_{1} \phi_{1}^{*}$. Then the perturbed metric tensor and electromagnetic field are given by the expressions

$$
\begin{gathered}
h_{a b}=2\left\{l_{a} l_{b}\left(\delta+3 \beta+\alpha^{*}-\tau\right) M_{1^{\prime}}+m_{a} m_{b}(D-\rho) M_{0^{\prime}}-l_{(a} m_{b)} \times\right. \\
\left.\left[\left(D-\rho+\rho^{*}\right) M_{1^{\prime}}+\left(\delta+3 \beta-\alpha^{*}-\tau-\pi^{*}\right) M_{0^{\prime}}\right]\right\}+ \text { c.c } \\
\hat{A}_{a}=\frac{1}{2}\left[l_{a}(\delta+2 \beta+\tau) \Psi_{E}-m_{a}^{*}(D+\rho) \Psi_{E}\right]+\text { c.c. }
\end{gathered}
$$

They satisfy the combined perturbation equations

$$
\begin{gathered}
\nabla^{c} \nabla_{(a} h_{b) c}-\frac{1}{2} \nabla_{c} \nabla^{c} h_{a b}-\frac{1}{2} \nabla_{a} \nabla_{b} h_{c}^{c}-\frac{1}{2} g_{a b}\left(\nabla^{c} \nabla^{d} h_{c d}-\nabla_{c} \nabla^{c} h_{d}^{d}\right. \\
\left.-\frac{1}{2} F_{c d} F^{c d} h_{e}^{e}+4 F^{c d} \nabla_{c} \hat{A}_{d}\right)-\frac{1}{2} F_{c d} F^{c d} h_{a b}-2 F_{a c} F_{b d} h^{c d}+4 F_{(a}^{c} \nabla_{b} b_{c}+4 F_{c(a} \nabla^{c} \hat{A}_{b)}=0
\end{gathered}
$$

and

$$
\nabla^{c} \nabla_{c} \hat{A}_{a}-\nabla^{c} \nabla_{a} \hat{A}_{c}-\nabla_{b}\left(F_{c a} h^{b c}\right)-F_{b c} \nabla^{b} h_{a}^{c}+\frac{1}{2} F_{b a} \nabla^{b} h_{c}^{c}=0,
$$

where $F_{c d}=\nabla_{c} A_{d}-\nabla_{d} A_{c}$ is the electromagnetic field of the background spacetime. $\left(\nabla_{a}\right.$ is the normal covariant derivative associated with a curved spacetime). The equations for the functions $\Psi_{G}, \Psi_{E}, M_{1^{\prime}}$ and $M_{0^{\prime}}$ are clearly quite difficult to solve. If there is no charge then these equations can be readily solved giving the solutions (5). If there is no rotation Torres del Castillo [9] has demonstrated their equivalence to the known solutions [1].

Very interesting is that the potential equations admit a nonobvious symmetry operator. This is a consequence of the following observations. Take a solution of the potential equations (8) and calculate the perturbed metric and electromagnetic field. These perturbed quantities can be used to calculate the perturbed components of the Weyl spinor $\Psi_{3}$ and $\Psi_{4}$ together with perturbed spin coefficents $\lambda$ and $\nu$. The combinations

$$
u_{3}=\frac{1}{4}\left(3 \Psi_{2} \phi_{2}-2 \Psi_{3} \phi_{1}\right), \quad u_{4}=-\Psi_{4} \phi_{1}, \quad v_{0}=\left(\delta^{*}+2 \alpha+3 \pi\right) \phi_{2}-2 \lambda \phi_{1},
$$




$$
v_{1}=(\Delta+2 \gamma+3 \mu) \phi_{2}-2 \nu \phi_{1}
$$

satisfy the equations

$$
\begin{gathered}
\left(\delta+3 \beta-\alpha^{*}+\pi^{*}\right) v_{0}-\left(D-\rho^{*}\right) v_{1}=-8 u_{3}, \\
\left(\delta^{*}+3 \pi+3 \alpha+\beta^{*}-\tau^{*}\right) v_{1}-\left(\Delta+3 \mu+3 \gamma+\mu^{*}-\gamma^{*}\right) v_{0}=2 u_{4}, \\
-4\left(\delta^{*}+2 \alpha+6 \pi\right) u_{3}+2(D-3 \rho) u_{4}=-v_{0} \Omega_{+} \\
-4(\Delta+2 \gamma+6 \mu) u_{3}+(\delta+4 \beta-3 \tau) u_{4}=-v_{1} \Omega_{-} .
\end{gathered}
$$

If we now write

$$
u_{3}=U_{3} \frac{e^{i(m \phi+\sigma t)}}{\sqrt{2} \bar{\rho}^{* 5}}, \quad u_{4}=U_{4} \frac{e^{i(m \phi+\sigma t)}}{\bar{\rho}^{* 6}}, \quad v_{0}=4 V_{0} \frac{e^{i(m \phi+\sigma t)}}{\bar{\rho}^{* 3}}, \quad v_{1}=V_{1} \frac{e^{i(m \phi+\sigma t)}}{\sqrt{2} \bar{\rho} \bar{\rho}^{* 3}}
$$

the resulting equations become

$$
L_{-1,2,-3} V_{0}-D_{1,0,-3} V_{1}=\frac{-2 \bar{\rho}}{\bar{\rho}^{* 2}} U_{3}, \quad L_{1,-1,3} V_{1}+\Delta D_{-1,-1,3} V_{0}=\frac{\bar{\rho}}{\bar{\rho}^{* 2}} U_{4},
$$

$L_{1,-1,3} U_{3}-D_{1,0,-3} U_{4}=2 \bar{\rho}^{* 3} \Omega_{+} V_{0}, \quad L_{-1,2,-3} U_{4}+\Delta D_{-1,-1,3} U_{3}=-2 \bar{\rho}^{* 3} \Omega_{-} V_{1}$.

If we make the identifications

$$
\bar{M}_{0^{\prime}}=\bar{\rho}^{* 3} v_{0}, \quad \bar{M}_{1^{\prime}}=\bar{\rho}^{* 3} v_{1}, \bar{\Psi}_{E}=\frac{2 \bar{\rho}^{* 3}}{\varphi_{1}} u_{3}, \quad \bar{\Psi}_{G}=\frac{\bar{\rho}^{* 3}}{\varphi_{1}} u_{4}
$$

then $\bar{M}_{0^{\prime}}, \bar{M}_{1^{\prime}}, \bar{\Psi}_{E}$ and $\bar{\Psi}_{G}$ satisfy the potential equations. Indeed it is possible to write this transformation in quite explicit form. The spin coefficents can be written

$$
\begin{gathered}
\lambda=-\frac{1}{2}\left[\frac{1}{\sqrt{2} \bar{\rho}^{*}} L_{-1,2,0}^{+} D_{0,1,-1} M_{1^{\prime}}+\frac{1}{2 \rho^{2}}\left\{L_{-1,2,0}^{+} L_{1,1,2}+2 \Delta D_{0,0,0}^{+} D_{0,1,0}\right\} M_{0^{\prime}}\right] \\
\nu=\frac{1}{4 \rho^{2}}\left[\left(2 L_{0,2,2}^{+} L_{1,1,-1}+\Delta D_{-1,0,1}^{+} D_{0,1,-1}\right) M_{1^{\prime}}+\frac{1}{\bar{\rho}} \Delta D_{-1,0,0}^{+} L_{2,1,2} M_{0^{\prime}}\right] \\
\phi_{2}=\frac{1}{4 \rho^{2}}\left[L_{-1,2,2}^{+} L_{1,-1,0}+\Delta D_{0,0,0}^{+} D_{0,-1,0}\right] \Psi_{E} .
\end{gathered}
$$

With these expressions $v_{0}$ and $v_{1}$ can be found using (11), i.e.,

$$
v_{0}=\frac{1}{\sqrt{2} \bar{\rho}} L_{-1,5,0} \phi_{2}-2 \lambda \phi_{1}, \quad v_{1}=\frac{-\Delta}{2 \rho^{2}} D_{1,-5,0}^{+} \phi_{2}-2 \nu \phi_{1} .
$$


Identifying $v_{0}$ and $v_{1}$ with $v_{0}=\bar{M}_{0^{\prime}} / \bar{\rho}^{* 3}$ and $v_{1}=\bar{M}_{1^{\prime}} / \bar{\rho}^{* 3}$ we can deduce expressions for $\bar{\Psi}_{E}$ and $\bar{\Psi}_{G}$ from the first two of the potential equations which are valid for $\bar{M}_{0^{\prime}}, \bar{M}_{1^{\prime}}, \bar{\Psi}_{E}$ and $\bar{\Psi}_{G}$. In the case of a spherically symmetric charged black hole this third order operator has been given by Torres del Castillo [9]. The key question is, how might the potential equations be solved? If there is no charge we can decouple them and produce the Teukolsky equations already discussed. If we repeat this procedure when the black hole has charge we obtain

$$
\begin{aligned}
& \left(\Delta D_{1,0,0} D_{-1,0,0}^{+}+L_{2,0,0}^{+} L_{-1,0,0}+6 i \sigma \bar{\rho}\right) U_{3}=-2 q^{2} \bar{\rho}^{* 2}\left[D_{0,2,0}\left(\frac{V_{1}}{\bar{\rho}}\right)+L_{2,2,0}\left(\frac{V_{0}}{\bar{\rho}}\right)\right] \\
& \left(\Delta D_{-1,0,0}^{+} D_{0,0,0}+L_{-1,0,0} L_{2,0,0}^{+}+6 i \sigma \bar{\rho}\right) U_{4}=2 q^{2} \bar{\rho}^{* 2}\left[-L_{-1,2,0}\left(\frac{V_{1}}{\bar{\rho}}\right)+\Delta D_{-1,2,0}^{+}\left(\frac{V_{0}}{\bar{\rho}}\right)\right]
\end{aligned}
$$

These equations can be turned into a pair of integral differential equations. Indeed if we can find the Green's functions which satisfy

$$
\begin{aligned}
& \left(\Delta D_{-1,0,0}^{+} D_{0,0,0}+L_{-1,0,0} L_{2,0,0}^{+}+6 i \sigma \bar{\rho}\right) G_{-2}\left(r, \theta: r^{\prime}, \theta^{\prime}\right)=\delta\left(r-r^{\prime}\right) \delta\left(\theta-\theta^{\prime}\right), \\
& \left(\Delta D_{1,0,0} D_{-1,0,0}^{+}+L_{2,0,0}^{+} L_{-1,0,0}+6 i \sigma \bar{\rho}\right) G_{-1}\left(r, \theta: r^{\prime}, \theta^{\prime}\right)=\delta\left(r-r^{\prime}\right) \delta\left(\theta-\theta^{\prime}\right),
\end{aligned}
$$

equations (14) can be inverted and solved for $U_{3}$ and $U_{4}$ via

$$
\begin{aligned}
& U_{3}=U_{3}^{(0)}-2 q^{2} \int_{r_{+}}^{\infty} \int_{0}^{\pi} G_{-1}\left(r, \theta: r^{\prime}, \theta^{\prime}\right) \bar{\rho}^{\prime * 2}\left[D_{0,2,0}^{\prime}\left(\frac{V_{1}^{\prime}}{\bar{\rho}^{\prime}}\right)+L_{2,2,0}^{\prime}\left(\frac{V_{0}^{\prime}}{\bar{\rho}^{\prime}}\right)\right] \frac{\sin \theta^{\prime}}{\Delta^{\prime}} d r^{\prime} d \theta^{\prime} \\
& U_{4}=U_{4}^{(0)}+2 q^{2} \int_{r_{+}}^{\infty} \int_{0}^{\pi} G_{-2}\left(r, \theta: r^{\prime}, \theta^{\prime}\right){\overline{\rho^{\prime}}}^{* 2}\left[-L_{-1,2,0}^{\prime}\left(\frac{V_{1}^{\prime}}{\bar{\rho}^{\prime}}\right)+\Delta^{\prime}{D^{\prime+}}_{-1,2,0}\left(\frac{V_{0}^{\prime}}{\bar{\rho}^{\prime}}\right)\right] \frac{\sin \theta^{\prime}}{\Delta^{\prime 2}} d r^{\prime} d \theta^{\prime},
\end{aligned}
$$

where $r_{+}=M+\sqrt{M^{2}-a^{2}-q^{2}}$ is the radius of the event horizon, which is a convenient physical boundary. The resulting integral differential equations are

$$
\begin{gathered}
-2 q^{2} \int_{r_{+}}^{\infty} \int_{0}^{\pi} \Delta D_{-1,3,0}^{+} G_{2}\left(r, \theta: r^{\prime}, \theta^{\prime}\right)\left[D_{0,0,0}^{\prime}\left(\frac{V_{1}^{\prime}\left(\overline{\rho^{\prime *}}\right)^{2}}{\bar{\rho}^{\prime}}\right)+L_{2,0,0}^{\prime}\left(\frac{V_{0}^{\prime}\left({\overline{\rho^{\prime}}}^{*}\right)^{2}}{\bar{\rho}^{\prime}}\right)\right] \frac{\sin \theta^{\prime}}{\Delta^{\prime 2}} d r^{\prime} d \theta^{\prime}+ \\
2 q^{2} \int_{r_{+}}^{\infty} \int_{0}^{\pi} L_{2,-3,0}^{+} G_{1}\left(r, \theta: r^{\prime}, \theta^{\prime}\right)\left[-L_{1,0,0}^{\prime}\left(\frac{V_{1}^{\prime}\left({\overline{\rho^{\prime}}}^{*}\right)^{2}}{\bar{\rho}^{\prime}}\right)+\Delta^{\prime} D_{-1,0,0}^{+}\left(\frac{V_{0}^{\prime}\left({\overline{\rho^{\prime}}}^{*}\right)^{2}}{\bar{\rho}^{\prime}}\right)\right] \frac{\sin \theta^{\prime}}{\Delta^{\prime}} d r^{\prime} d \theta^{\prime} \\
+\Delta D_{-1,3,0}^{+} U_{3}^{(0)}+L_{3,-3,0}^{+} U_{4}^{(0)}=-2 V_{1} \bar{\rho}^{* 3} \Omega_{-},
\end{gathered}
$$


and

$$
\begin{gathered}
-2 q^{2} \int_{r_{+}}^{\infty} \int_{0}^{\pi} L_{-1,3,0} G_{2}\left(r, \theta: r^{\prime}, \theta^{\prime}\right)\left[D_{0,0,0}^{\prime}\left(\frac{V_{1}^{\prime}\left({\overline{\rho^{\prime}}}^{*}\right)^{2}}{\bar{\rho}^{\prime}}\right)+L_{2,0,0}^{\prime}\left(\frac{V_{0}^{\prime}\left({\overline{\rho^{\prime}}}^{*}\right)^{2}}{\bar{\rho}^{\prime}}\right)\right] \frac{\sin \theta^{\prime}}{\Delta^{\prime 2}} d r^{\prime} d \theta^{\prime} \\
-2 q^{2} \int_{r_{+}}^{\infty} \int_{0}^{\pi} D_{0,-3,0} G_{1}\left(r, \theta: r^{\prime}, \theta^{\prime}\right)\left[-L_{-1,0,0}^{\prime}\left(\frac{V_{1}^{\prime}\left({\overline{\rho^{\prime}}}^{*}\right)^{2}}{\bar{\rho}^{\prime}}\right)+\right. \\
\left.\Delta^{\prime} D_{-1,0,0}^{+}\left(\frac{V_{0}^{\prime}\left({\overline{\rho^{\prime}}}^{*}\right)^{2}}{\bar{\rho}^{\prime}}\right)\right] \frac{\sin \theta^{\prime}}{\Delta^{\prime}} d r^{\prime} d \theta^{\prime}+L_{-1,3,0} U_{3}^{(0)}-D_{0,-3,0} U_{4}^{(0)}=2 V_{1} \bar{\rho}^{* 3} \Omega_{+} .
\end{gathered}
$$

These two equations can be iterated to solve for the functions $U_{3}, U_{4}, V_{1}$ and $V_{2}$. Indeed the Green's functions can be written in the form

$$
G_{s}\left(r, \theta: r^{\prime}, \theta^{\prime}\right)=\sum_{n=1}^{\infty} G_{s}^{n}\left(r, r^{\prime}\right) S_{s, \lambda_{n}}(\theta) S_{s, \lambda_{n}}\left(\theta^{\prime}\right)
$$

where

$$
G_{s}^{n}\left(r, r^{\prime}\right)=\left[\begin{array}{ll}
R_{s, \lambda_{n}}^{(1)}(r) R_{s, \lambda_{n}}^{(2)}\left(r^{\prime}\right) \Delta^{\prime s}, & r>r^{\prime} \\
R_{s, \lambda_{n}}^{(2)}(r) R_{s, \lambda_{n}}^{(1)}\left(r^{\prime}\right) \Delta^{\prime s}, & r<r^{\prime}
\end{array}\right.
$$

for suitable radial Teukolsky functions $R^{(1)}$ and $R^{(2)}$. To determine which functions are chosen we need to look at the asymptotic behaviour at both infinity and the event horizon. To do this it is convenient to introduce the radial variable $r^{*}$ defined by

$$
\frac{d r^{*}}{d r}=\frac{r^{2}+a^{2}}{\Delta}
$$

The choice of $r^{*}$ is convenient since $r^{*} \rightarrow \infty$ as $r \rightarrow \infty$ and $r^{*} \rightarrow-\infty$ as $r \rightarrow r_{+}$(the boundary of the event horizon). Indeed if we consider instead the functions $Y_{s}=\Delta^{s / 2}\left(r^{2}+a^{2}\right)^{1 / 2} R_{s}$ then the asymptotic behaviour of these Teukolsky functions is

$$
Y_{-1} \approx A_{1} r^{-1} e^{i \sigma r^{*}}+A_{2} r e^{-i \sigma r^{*}}, \quad Y_{-2} \approx B_{1} r^{-2} e^{i \sigma r^{*}}+B_{2} r^{2} e^{-i \sigma r^{*}} .
$$

Assuming the $t$ dependence $e^{i \sigma t}$ we see that these solutions correspond to ingoing and outgoing waves. Similarly as the event horizon is approached we obtain the asymptotic behaviour

$$
Y_{-1} \approx C_{1} \Delta^{1 / 2} e^{i\left(\sigma+m \sigma_{+}\right) r^{*}}+C_{2} \Delta^{-1 / 2} e^{-i\left(\sigma+m \sigma_{+}\right) r^{*}}
$$




$$
Y_{-2} \approx D_{1} \Delta e^{i\left(\sigma+m \sigma_{+}\right) r^{*}}+D_{2} \Delta^{-1} e^{-i\left(\sigma+m \sigma_{+}\right) r^{*}},
$$

where $\sigma_{+}=\frac{a}{2 M r_{+}-q^{2}}$. These solutions also represent ingoing and outgoing waves at the horizon. As one would expect,the boundary conditions assumed for the Green's functions must depend on the problem under consideration. There are basically two types of problems we might consider:

[I]. A plane wave incident from $r^{*}=\infty$. We would then expect to have reflected waves at $r^{*}=\infty$ and transmitted waves at $r^{*}=-\infty$. For a full discussion of scattering in the vicinity of black holes see [10].

[II]. Alternatively, if perturbations of a black hole were being investigated one would expect outgoing waves at the event horizon $r^{*}=-\infty$, transmitted outgoing waves at $r^{*}=\infty$ and reflected ingoing waves at the event horizon $r^{*}=-\infty$. In this case we seek solutions $Y_{-2}$ which satisfy $Y_{-2} \approx B_{2} r^{2} e^{-i \sigma r^{*}}$ as $r^{*} \rightarrow \infty$ and $Y_{-2} \approx D_{1} \Delta e^{i\left(\sigma+m \sigma_{+}\right) r^{*}}+D_{2} \Delta^{-1} e^{-i\left(\sigma+m \sigma_{+}\right) r^{*}}$ as $r^{*} \rightarrow-\infty$.

If we adopt an iterative procedure using these integral equations then a suitable first approximant for the functions $V_{0}$ and $V_{1}$ is

$$
\begin{gathered}
V_{0}=-\frac{1}{2 \bar{\rho}^{* 3} \Omega_{+}}\left(L_{-1,3,0} R_{-1} S_{-1}-D_{0,-3,0} R_{-2} S_{-2}\right), \\
V_{1}=-\frac{1}{2 \bar{\rho}^{* 3} \Omega_{-}}\left(\Delta D_{-1,3,0} R_{-1} S_{-1}+L_{2,-3,0}^{+} R_{-2} S_{-2}\right) .
\end{gathered}
$$

This choice gives the potential functions $M_{0^{\prime}}$ and $M_{1^{\prime}}$, respectively. From the potential equations one can then determine the first approximate values of $\Psi_{E}$ and $\Psi_{G}$. Indeed there are two possible approximants possible at the zeroth level.

$[\mathrm{I}]$

$$
\begin{gathered}
M_{0^{\prime}}=\frac{-q}{2 \Omega_{+}} L_{-1,3,0} R_{-1} S_{-1}, \quad M_{1^{\prime}}=\frac{-q}{2 \Omega_{-}} \Delta D_{-1,3,0} R_{-1} S_{-1}, \\
\Psi_{E}=2 \sqrt{2} R_{-1} S_{-1}, \quad \Psi_{G}=0 .
\end{gathered}
$$

These solutions correspond to a combination of electromagnetic waves and charge induced gravitational waves. The expressions for the electromagnetic vector potential and the perturbed metric are

$$
A_{a}=\left\{l_{a} \frac{1}{\bar{\rho}} L_{1,-1,0}-m_{a} \sqrt{2} D_{0,-1,0}\right\} R_{-1} S_{-1},
$$




$$
\begin{gathered}
h_{a b}=\left\{l_{a} l_{b} \frac{(-q)}{\sqrt{2} \bar{\rho} \Omega_{-}^{2}}\left[3 \Psi_{2} L_{1,4,-1}-2 \Phi_{11} L_{1,6,-3}\right] \Delta D_{-1,3,0}+m_{a} m_{b} \frac{(-q)}{\Omega_{+}^{2}}\left[3 \Psi_{2} D_{0,4,0}\right.\right. \\
\left.+2 \Phi_{11} D_{0,6,2}\right] L_{-1,3,0}+l_{(a} m_{b)}\left(\frac{q}{\Omega_{-}^{2}}\left[3 \Psi_{2} D_{0,4,-1}-2 \Phi_{11} D_{0,0,1}\right] \Delta D_{-1,3,0}+\right. \\
\left.\left.\frac{q}{2 \bar{\rho} \Omega_{+}^{2}}\left[3 \Psi_{2} L_{2,4,2}+2 \Phi_{11} L_{2,0,0}\right] L_{-1,3,0}\right)\right\} R_{-1} S_{-1} .
\end{gathered}
$$

$[\mathrm{II}]$

$$
\begin{gathered}
M_{0^{\prime}}=\frac{-1}{2 \Omega_{+}} D_{0,-3,0} R_{-2} S_{-2}, \quad M_{1^{\prime}}=\frac{-1}{2 \Omega_{-}} L_{2,-3,0}^{+} R_{-2} S_{-2}, \\
\Psi_{E}=0, \quad \Psi_{G}=\frac{2}{q \bar{\rho}^{*}} R_{-2} S_{-2} .
\end{gathered}
$$

These solutions correspond to pure gravitational waves to this level of approximation. The expressions for the perturbed metric tensor are

$$
\begin{gathered}
h_{a b}=\left\{l_{a} l_{b} \frac{(-1)}{\sqrt{2} \bar{\rho} \Omega_{-}^{2}}\left[3 \Psi_{2} L_{1,4,-1}-2 \Phi_{11} L_{1,6,-3}\right] L_{2,-3,0}^{+}+\right. \\
m_{a} m_{b} \frac{(-1)}{\Omega_{+}^{2}}\left[3 \Psi_{2} D_{0,4,0}+2 \Phi_{11} D_{0,6,2}\right] D_{0,-3,0}+l_{(a} m_{b)}\left(\frac { 1 } { \Omega _ { - } ^ { 2 } } \left[3 \Psi_{2} D_{0,4,-1}\right.\right. \\
\left.\left.\left.-2 \Phi_{11} D_{0,0,1}\right] L_{2,-3,0}^{+}+\frac{1}{2 \bar{\rho} \Omega_{+}^{2}}\left[3 \Psi_{2} L_{2,4,2}+2 \Phi_{11} L_{2,0,0}\right] D_{0,-3,0}\right)\right\} R_{-2} S_{-2} .
\end{gathered}
$$

\section{Concluding remarks}

We have seen how special functions of Heun type occur when we consider the perturbation problem for a Kerr Newman black hole. The treatment of perturbations of black holes with no charge can be solved in terms of Heun functions. Why do these methods of solution work? If we go back to the result of Teukolsky one would naturally ask what the significance of the separation constant might be. A clue is provided in the study of free motion of a particle along a geodesic path in Kerr Newman space time. Indeed it is found that in addition to the obvious axial and time translation symmetry of the space time there is another constant of the motion that behaves like angular momentum. This crucial property of the Kerr Newman space time 
is related to the existence of a Killing Yano tensor. This is a skew symmetric tensor $K_{\mu \nu}$ which satisfies the Killing Yano equations

$$
\nabla_{(\mu} K_{\nu) \sigma}=0, \quad K_{(\mu \nu)}=0 .
$$

Relative to our standard tetrad the only nonzero components are

$$
K_{i j} m^{i} m^{j *}=r, \quad K_{i j} l^{i} n^{j}=i a \cos \theta .
$$

An important consequence is that $K_{\mu \nu}=K_{\mu \sigma} K_{\nu}^{\sigma}$ is a Killing tensor. This means that a constant of the geodesic motion can be constructed in the form $C=K^{\mu \nu} p_{\mu} p_{\nu}$. The existence of this constant of the motion was determined by Carter in his original investigations [11] of the separability properties of electro vacuum space times. The presence of the Killing Yano tensor enables the separation constant to be characterised in an invariant way. Indeed in terms of the solution of (5) found by Cohen and Kegeles there is an operator characterisation of the separation constant $\lambda$ in the form

$$
K^{\mu \nu} \nabla_{\mu} \nabla_{\nu} h_{\sigma \tau}+B_{\sigma \tau}^{\rho \epsilon \delta} \nabla_{\rho} h_{\epsilon \delta}+C_{\sigma \tau}^{\epsilon \delta} h_{\epsilon \delta}=\lambda h_{\sigma \tau} .
$$

The role of the Killing Yano tensor in solving wave equations corresponding to spin 1 and spin $1 / 2$ is also crucial [4]. One of the interesting theoretical questions is exactly how the existence of a Killing Yano tensor determines many of the properties we have described.

\section{References}

[1] S. Chandrasekhar. The Mathematical Theory of Black Holes. Oxford University Press, Oxford, 1983.

[2] J.M. Cohen \& L.S. Kegeles. Constructive procedure for perturbations of spacetimes. Phys.Rev. D19, 1641, (1979).

[3] S. Teukolsky. Perturbations of a rotating black hole. 1. Fundamental equations for gravitational, electromagnetic and neutrino field perturbations. Astr.Phys.J. 185, 635, (1973).

[4] E. G. Kalnins, W. Miller Jr. and G. C. Williams. Recent advances in the use of separation of variables methods in general relativity. In Classical General Relativity edited by S. Chandrasekhar. Oxford University Press, Oxford, 1993. 
[5] L.P. Eisenhart. Riemannian Geometry, Princeton University Press, Princeton, 1964.

[6] E.D. Fackerell and R.D. Crossman. Spin weighted angular spheroidal functions. J.Math.Phys. 18, 1849, (1977).

[7] A. Ronveaux, editor. Heun's differential equations. Oxford University Press, Oxford, 1995.

[8] G. F. Torres del Castillo. Complex potentials for coupled gravitational and electromagnetic perturbations. Classical and Quantum Gravity 4, 1011, (1987).

[9] G. F. Torres del Castillo. Gravitational and electromagnetic perturbations of the Reissner-Nordstrom solution. Classical and Quantum Gravity 4, 1133, (1987).

[10] J. A. Futterman, F. A. Handler and R. A. Matzner. Scattering from Black Holes. Cambridge University Press, Cambridge, 1988.

[11] B. Carter. Hamilton Jacobi and Schrödinger separable solutions of Einstein's equations. Phys.Rev. 10, 280, (1968). 